\title{
Bridging adaptive learning and desired natural resource management outcomes: insights from Australian planners
}

\begin{abstract}
Natural resource management (NRM) has been increasingly guided by governance arrangements seeking less centralised and hierarchical and more integrated and adaptive approaches to achieve desired social-ecological outcomes. Successful implementation of these approaches requires adaptive learning which entails the application of individual, institutional and social learning to adaptive co-management. This paper proposes and validates a conceptual model that identifies components of adaptive learning and their relationships with desired NRM outcomes. Supported by on-ground experience of Australian NRM planners, it discusses three key insights to enable bridging between adaptive learning and NRM outcomes: changing focus away from economic-efficiency culture, supporting learning and knowledge exchange structures, and reinventing practice.
\end{abstract}

Key words: adaptive co-management, social learning, environment, institutional change, planning

\section{Introduction}

Natural resource management (NRM) is a challenging task involving multiple actors attempting to address complex and dynamic environmental problems (van der Wal et al. 2014). In the Australian context, this challenge is further compounded by ongoing institutional changes (e.g. politically driven planning reforms, organisational restructuring, corporatisation of NRM) and reductions in funding affecting NRM organisations (Vogel 2013). As the pressure mounts onto NRM organisations to become more efficient and, to some extent, profitable and self-funded, much of their 
time is now spent in securing their existence (Vella et al. 2015). This leads to competition with the environmental outcomes they are deemed to be achieving as their primary function. As planners working for NRM organisations are directly affected by these challenges, the problem that this paper seeks to investigate is the role of learning in assisting them to better carry out their work and ultimately achieve the desired NRM outcomes - which is the core business of their organisations.

Theories and concepts related to what learning is and how it occurs have emerged in several disciplines and fields of knowledge such as psychology, education and neuroscience (Plummer et al. 2007). However, this paper is underpinned by learning theories and concepts that have been identified to influence NRM processes, particularly social learning ${ }^{1}$ (Muro et al. 2008). Drawing on action research (Reason et al. 2006) involving planners from the Australian East Coast Custer of NRM organisations, the paper applies a conceptual model that explores how planners rate the extent to which various processes relating to NRM learning and identified learning enablers facilitate the achievement of NRM outcomes. These planners have a critical role within their organisations because they are responsible for the making and implementation of strategic plans; delivery of programs to better manage land, water and biodiversity assets; and continuing reporting of achievements often used to secure funding and investment in NRM. Thus, identifying what helps or impedes their learning is important as it can improve the NRM planning cycle overall and, importantly, assist planners to better perform their challenging roles.

\footnotetext{
${ }^{1}$ Social learning generally refers to learning that occurs through interaction and collaboration which leads to shared knowledge and understanding as well as change in attitudes and beliefs at both the individual and social units level (Baird et al. 2014, Newig et al. 2010, Reed et al. 2010).
} 
To this end, the paper first summarises the literature on processes facilitating learning for NRM based on which the conceptual model is proposed. It then describes the research approach, including the validation of the conceptual model with NRM planners, and results related to learning processes concerning NRM outcomes. Based on findings, it discusses key insights for bridging adaptive learning and NRM outcomes to build NRM organisation's capacity, and of their planners, to respond to environmental and institutional change. The paper concludes with some overall implications of the findings for the broader planning practice.

\section{Overview of processes relating to learning in NRM}

Planners working in the NRM sector are often bombarded with a myriad of theories and concepts relating to learning that attempt to guide their practice, making it complex and difficult to apply to on-ground activities (Armitage et al. 2008). Because NRM problems are complex and require a wide range of expertise and knowledge, many NRM learning theories and concepts can be broadly related back to two key strands of literature: (i) management approaches to NRM, and (ii) knowledge exchange structures.

Similar to worldwide trends, planning approaches to NRM in Australia are dominated by the adaptive management, co-management and adaptive co-management paradigms (Berkes 2009). These paradigms reflect the need to support evidencebased decision-making in an increased contested space involving NRM - that is, a plethora of conflicting stakeholder's interests and values concerning natural resources (Cvitanovic et al. 2016). However, there is no single framework for such paradigms, or agreed definition (Hasselman 2016), that planners can rely on. Generally, they follow agreed common components instead, including being a cyclical iterative process based on monitoring, evaluation, reporting and improvement (MERI) 
(Plummer 2009). Hence, learning associated with how to carry out any of these forms of management to achieve desired environmental outcomes becomes fundamental for planners at the forefront of their organisations. This need for learning has encouraged many planners in the NRM sector to join forces to carry out their challenging job by establishing knowledge exchange structures (Hobday et al. 2017).

Knowledge exchange involves all aspects of knowledge generation and application process, including its translation to inform on-ground actions (Cvitanovic et al. 2016). A model that describes the interface between knowledge production and application is found in the suite of activities known collectively as ' $K$ '' (Shaxson et al. 2012). While not exclusive to the NRM sector, these activities are important for both individual and organisational learning and include knowledge management, transfer, translation, exchange, brokering, and mobilisation. In the NRM sector, ' $\mathrm{K}^{\star}$ ' are often discussed within the context of knowledge translation enabled through boundary organisations (Crona et al. 2012, 2011, Berkes 2009), bridging organisations (Crona et al. 2012, Berkes 2009), and knowledge brokering (Bielak et al. 2008, Michaels 2009).

Knowledge exchange can be facilitated through communities of practice, inter-regional learning and organisational learning. Communities of practice are defined as a group of practitioners (in a particular field) increasing their knowledge and expertise through ongoing interaction (Wenger 1998, 2000). They also enable the sharing of tacit knowledge and practical experiences that are not otherwise documented (Ardichvili et al. 2003). Communities of practice are particularly relevant for the NRM sector because they enable learning to occur beyond a single organisation, and include individuals and societies learning as part of a complex adaptive system as the basis of environmental problem solving (Baird et al. 2014). Similarly, inter-regional learning (sometimes termed transnational learning) relies on learning networks (Hachmann 
2008). It differs from communities of practice in that the focus is generally regarded as being on the transfer of policy or management strategies between decision making organisations, the learning is manifest by behaviour change at the organisational level, and that the learning includes reflection on the broader institutional context (Hachmann 2008). In contrast, communities of practice focus on individual practices and learning by individual practitioners.

Elements of organisational learning are also understood to have a role in NRM, especially because of ongoing institutional changes NRM organisations are subject to (Lonsdale 2012). Organisational learning may be defined as a learning process that occurs within an organisation or company, particularly in relation to the goals, function, process and longevity of the organisation (De Geus 1988). The literature on organisational learning is most relevant to the business of NRM - that is, the functioning of the NRM organisations themselves.

Challenges confronting planners working in the NRM sector are unlikely to subside as environmental degradation and demand for natural resources continue to rise (Hobday et al. 2017). Hence, understanding the ways in which individual and project-based learning is encoded in NRM organisations, and the ways in which they approach learning as organisations, could be instrumental in improving their effectiveness in enhancing their adaptive capacity.

\section{Developing the conceptual model}

Based on the processes relating to learning in NRM presented earlier, a conceptual model is proposed to identify their relationships with desired NRM outcomes. The conceptual model starts by taking adaptive co-management as the most advanced paradigm that is currently underpinning NRM in Australia and elsewhere (Berkes 2009). The model also assumes that adaptive co-management is driven by adaptive 
learning (Smith et al. 2009). Adaptive learning offers a big umbrella (see Figure 1) to assemble these varied learning processes and, therefore, is suitable to address complexity and uncertainty in NRM (Reed et al. 2006, Davidson-Hunt et al. 2003), including those prompted by environmental and institutional changes (Pahl-Wostl 2007).

\section{[insert Figure 1 near here]}

There are several inherent aspects to adaptive learning that support its usefulness to inform NRM decision-making. For example, adaptive learning can bring together bottom-up and top-down approaches to deliver best practice for NRM (Reed et al. 2006). It can assist in building knowledge based on feedback loops - learning from mistakes and successes of implemented actions (Davidson-Hunt et al. 2003). It also promotes approaches that facilitate communication and knowledge sharing within and between organisations in the development of adaptive strategies that deal with changing social and environmental conditions (Reed et al. 2009).

Table 1 summarises the several learning processes identified in the literature that have relevance for NRM, including how these learning processes may relate to outcomes sought by NRM organisations. Although program auditing has not been extensively discussed in the NRM literature as a learning process (Cook et al. 2016, Viegas et al. 2013), it has been added to the conceptual model because it is an essential part of evaluation in a NRM organisation, particularly for programs funded by governments that must show efficiency and accountability which is the Australian case (Vella et al. 2015). The conceptual model (Figure 2) synthesises this information to:

- identify key concepts and terminology in the NRM learning literature

- identify how these concepts relate to NRM practice

- identify potential NRM learning outcomes 
- assist in identifying processes and activities that promote different types of NRM learning outcomes.

[insert Table 1 near here]

[insert Figure 2 near here]

\section{Research approach, methods, and process insights}

\section{Case study: the East Coast Cluster}

The delivery of NRM plans and outcomes in Australia predominately occurs at the regional scale (Lockwood et al. 2009). Currently, there are 56 regional natural resource organisations in Australia (Australian Government 2014), which were grouped into eight clusters for the purpose of incorporating climate change adaptation information into their regional plans. This paper focuses on the East Coast Cluster, comprising six regional organisations on the east coast of the states of Queensland and New South Wales (NSW) (see Figure 3). Governance arrangements for NRM vary between the two states. In QLD, NRM organisations are community based, nonstatutory and adopt different governance models, although they are generally governed by a community-based board comprising industry representatives and expert members (Vella et al. 2015). In NSW, NRM organisations are statutory and follow a state-wide governance model - i.e., they are overseen by an independent local board consisting of government representatives and community members who are elected by ratepayers in each region (NSW Government 2013).

[insert Figure 3 near here]

A research consortium involving four research institutions and two state government agencies was created to carry out the Climate Change Adaptation for NRM in East 
Coast Australia Project (thereafter referred to as the project). This three-year project was designed to foster and support an effective community of practice for climate change adaptation within the East Coast Cluster through the primary engagement of their planners. Each member of the research consortium was responsible for delivering research outputs aimed to increase the Cluster's capacity for adaptation to climate change through enhancements in knowledge and skills, and establishment of long-term collaborations between consortium partners and NRM organisations. This paper refers to the work carried out by one of the research consortium members responsible for the engagement activities with the Cluster planners.

NRM issues vary across Australia and there is corresponding variation between the NRM organisations in their organisational practices, legislative frameworks and foci (Robins et al. 2007). The area and population of NRM regions varies widely, as does access to information, priority issues, state legislation, organisational structure and connections with government and research institutions (Robins et al. 2007). While findings from this study may not be transferable or representative of other regions or clusters, they are still useful for NRM organisations because they provide in-depth insights into the issues affecting the achievement of desired NRM outcomes. Findings may also useful for the planning practice, especially for planners working in less resourced governments that have limited capacity to deal with complex and contested environmental issues.

\section{Research design}

The QLD based NRM organisations of the East Coast Cluster already operated a Planners Working Group (PWG) prior to the project which served as the basis for the engagement activities. The PWG comprised the main method for information sharing and capacity building throughout the project. NRM organisations in the Cluster 
employed between one or two planners to develop and implement their NRM plans. It was this cohort of planners that participated with the authors in the iterative activities of the PWG.

Adopting an action research approach (Reason et al. 2006), engagement activities involving the PWG were held between May 2013 and June 2015 and comprised a series of five two-day workshops and ongoing discussions via shared mailing lists. All two-day workshops followed the same structure by allocating consortium based research activities on the first day and PWG activities on the second day. The first day of the two-day workshops played an important role in identifying the needs of the NRM organisations as a focus for research as well as the usability and uptake of research outputs prepared by consortium partners. These workshops included presentations and discussions from research consortium partners as well as external research organisations (including researchers from other clusters and national project teams). Presentations were interactive to enable workshop participants to clarify any doubts about research outputs and provide feedback to researchers related to the best format research outputs should take to maximise their applicability in their organisation's planning and decision-making process.

The second day of the two-day workshops was exclusively dedicated to the PWG to enable planners to discuss the challenges encountered in the day-to-day operation of their organisations, especially the ongoing institutional changes caused by uncertainty in funding, regulatory frameworks, organisational restructuring and subsequent staff churn, and applicability of research outputs prepared by research consortium partners in their planning and decision-making activities. These PWG dedicated workshops and shared mailing lists were instrumental in facilitating interactions among the planners as a community of practice. 
Discussions held at all workshops were essential in providing information relative to desired NRM outcomes these organisations sought to achieve. In particular, the challenges faced by members of the PWG clearly indicated the need for improved learning strategies to be adopted by their organisations. For example, half way through the project, the NSW based organisations went through a full restructuring process which resulted in staff churn and changes to their core business. Further, only two of the six organisations were able to effectively employ the same planner for the whole duration of the project. Discussions held at each series of workshops were carried out in plenaries and small group activities. In both cases, discussions were captured through workshop notes and worksheets and then summarised as workshop reports that were subsequently shared with workshop participants for verification.

\section{Process insights and validation of the conceptual model}

The conceptual model proposed earlier (Figure 3) was informed by and refined through engagement activities carried out with the PWG. The validation of the conceptual model draws on data obtained at the second day of the fourth series of workshops held in November 2014 which was attended by seven PWG members representing five NRM organisations. At that workshop, participants were presented with the proposed conceptual model to identify and discuss the relationship between the different processes relating to learning and NRM outcomes, including the types of activities that enabled learning to occur. All seven participants were responsible for developing and implementing NRM plans for their organisations and their feedback on the conceptual model was directly informed by their on-ground experience in carrying out this important task.

The validation process started with a whole group discussion about what adaptive capacity meant in the NRM context with a focus on the role of science informing policy, 
stakeholder engagement, organisational learning and improved governance. Participants were also prompted to discuss what learning is in the NRM context with the assumption that learning is already a focus of NRM activity but there is relatively low awareness of how it is occurring, including the multiple learning opportunities within existing practices. The conceptual model was then presented to participants as a potential framework that could be used to improve understanding of the relationships between the core activities the Cluster organisations engaged in and different learning processes relevant to NRM extracted from the literature. It was highlighted that the purpose of providing such a framework was to assist NRM organisations in assessing the range of learning activities that they undertake, in relation to the relevant learning goals and outcomes, and to help in identifying processes and practices that promote specific types of learning.

Participants were then asked to test the conceptual model and identify any issues, overlaps, redundancies and gaps. This test was done through a series of three exercises initially carried out individually with subsequent group discussion at the end of each session. Results from this test are summarised in Figure 4 and Table 2.

[insert Figure 4 near here]

[insert table 2 near here]

In the first exercise participants were asked to identify the learning processes that best aligned with the set of NRM outcomes. To do this, they were given a table listing the learning processes extracted from the literature (i.e., knowledge translation, adaptive co-management, MERI, program auditing, inter-regional learning, communities of practice and organisational learning) and a blank version of the conceptual model shown in Figure 2 in which only the list of outcomes was included (i.e., evidence-based 
policy, collective action, ecosystem health, efficient resource use, effective institutions, best practice, and effective organisation).

Many of the workshop participants commented that it was difficult to link just one learning process as many of the outcomes were overlapping. Participants indicated that the separation of best practice from others is problematic as best practice can emerge from a range of learning processes; the effectiveness of a particular investment can drive funding and also improve practice to demonstrate how the investment is being effective on the ground; and adaptive co-management was considered to have a direct relationship with evidence-based policy, ecosystem health, effective organisations, best practice in addition to collective action.

Participants also noted problems with some of the terminology in the conceptual model. They indicated that evidence based decision was the adequate terminology to what they seek to achieve as opposed to evidence based policy. The term effective institutions was updated to effective organisation (multiple) to represent NRM organisations in general, whereas effective organisation (individual) represented each of the NRM organisations represented in the PWG.

In the second exercise, participants recorded which were their current practices and activities (see enablers in Figure 4), and the reasons and motivations (see reasons in Figure 4) for doing it for each outcome. Enablers associated with adaptive comanagement primarily included structured and spontaneous engagement activities such as roundtables and informal review processes. Other established learning processes such as program auditing, although being resource intensive, was found to have the least potential for contributing to achieving multiple NRM outcomes. Excessive micro-management reporting systems and requirements were identified as being learning impediments rather than enablers. Enablers characterised by some 
degree of collaboration between organisations, planners and/or stakeholders were identified to support several NRM outcomes. Examples included project partnerships with institutional and non-institutional stakeholders for supporting collective action; collaboration with other organisations to achieve synergies based on achieving common objectives for supporting effective resource use; and cross-regional teams developing policies, plans and practices for supporting best practice. Review processes were identified to contribute to achieving several NRM outcomes such as evidence-based decisions, collective action and effective individual organisations.

With respect to the reasons and motivations behind seeking to achieve NRM outcomes, participants noted funding/ resource issues affecting these organisations. In particular, for several NRM outcomes the requirement to justify investment in particular areas was identified as the main driver to report achievements rather than actual on-ground efficiency of activities.

In the last exercise, participants ranked the importance of the different learning processes for their respective organisations in terms of their current level of importance, extent of input, impact on achieving outcomes, potential in achieving longterm outcomes, and the extent to which organisational processes need to change to achieve these outcomes (see Table 2). Ranking ranged from 1 (low/ not much/ not very important) to 5 (high/ a lot/ very important).

Adaptive co-management was ranked highly across all fields except needed organisational change, indicating that it is currently very important, is given a lot of effort, and has high potential in achieving future outcomes, but there is little room for improvement. MERI was rated as having the least potential for achieving future outcomes, but was also ranked as having currently high importance in the organisation and receiving a lot of effort. Program auditing was ranked as having the lowest 
potential for the future. Knowledge translation, communities of practice and organisational learning were all rated as having the highest need for organisational change, and high potential for future impacts, but are currently of low importance in the organisations and receive relatively little effort. Inter-regional learning was similar, but was rated as having lower need for organisational change.

\section{Bridging NRM adaptive learning processes and desired outcomes}

As the future is essentially unknowable, learning concerning NRM is critical to build adaptive capacity (Crona et al. 2012). This is particularly relevant because many NRM organisations are already stretched in their capacity to manage existing land use pressures and competition for resources (expanding urbanisation, water extraction etc.) which are likely to be exacerbated under climate change (Hobday et al. 2017). In this context, adaptive capacity refers to what supports knowledge exchange to occur within and between organisations and individuals, and therefore enhance planners' capability to carry out their jobs with competence considering the dynamic and complex nature of environmental issues they have to manage (Cvitanovic et al. 2016).

Given the current focus in NRM on adaptive co-management, learning comprises a core activity in most NRM organisations. There are multiple opportunities for learning, some of which are formally recognised and explicitly formulated as such, while others ostensibly have other purposes but have potential learning outcomes. While there is a multitude of learning processes that are currently occurring in NRM, the validation of the proposed conceptual model raised three key insights about interconnected areas that need improving: changing focus away from economic-efficiency culture; supporting knowledge exchange structures; and reinventing NRM practice. 


\section{Changing focus away from economic-efficiency culture}

Both learning in NRM and NRM practice are influenced by governance contexts and institutional arrangements (Patterson 2016). In Australia, NRM governance contexts and institutional arrangements have changed significantly from a largely regulated, government driven approach until the 1990's to a more voluntary, collaborative, best practice approach over the last decades (Vella et al. 2015, Jacobson et al. 2014). Despite being heavily based on principles of adaptive governance, these changes did not result in improved on-ground NRM outcomes and the reduced amount of available funds for NRM increased competition between organisations (Vella et al. 2015, Gooch et al. 2009). For example, these changes resulted in reduced organisational funding leading to staff churn and ceasing of programmes, including successful ones (Tennent et al. 2012).

NRM organisations comprising the East Coast Cluster are no exception to this situation which is reflected in the results from the conceptual model validation test. Planners often referred to 'investment', 'managing limited resources', 'accountability', 'funding applications' as underlying reasons for learning in response to the existing culture focused on economic-efficiency that permeates their work. They also noted how program auditing does little to contribute to achieving multiple and/ or single NRM outcomes - a finding supported by a recent review of NRM in Australia which has shown that the delivery of measurable and tangible NRM outcomes has not been achieved to the desired extent (Clayton et al. 2011). The review suggests that improvements in natural resources led by NRM organisations have been dwarfed by impacts outside their control (such as large-scale land clearing due to mining, agricultural or urban expansion). 
There is a need to shift away from the current focus of economic-efficiency culture driving NRM funding to address environmental issues holistically (Jacobson et al. 2014), including recognising the multiple learning situations in NRM organisations' practice, and their features, and to explicitly link and integrate these with NRM outcomes. Based on systems thinking, innovation and learning can be inhibited by a reliance on models that assume outcomes can be reliably predicted, and that performance can be evaluated based on meeting these outcomes (Goold 2006, Chapman 2004). This does not necessarily imply moving away from successful program logic based, target-setting approaches (Chapman 2004). Rather, systems thinking calls for adding another level of abstraction by considering the wider context - in this case, reflection on the role of the NRM organisation in the broader governance system, and the functioning of the governance system itself. This involves adding at least two additional layers: (i) assessing the collective impact of the organisation and stakeholders in their region beyond economic rationality; and (ii) the collective impact of the NRM governance system as a whole, including building relationships and capacity through learning situations in NRM organisations' practice (Ryan et al. 2010).

\section{Supporting knowledge exchange structures}

Improving how learning can be maintained by NRM organisations is critical as challenging institutional conditions are unlikely to disappear under increased global demand for natural resources and political pressures on governments to facilitate their exploitation (Dietz et al. 2003). The collaborative nature of NRM means that social learning provides the underlying context for many of the interactions between NRM planners and stakeholders, and attention should be paid to power relations and trust issues underpinning collaborations (Levesque et al. 2016). Given the voluntary, collaborative connotation and reduced regulation to NRM practice in Australia, NRM 
organisations can only achieve their aims by working in partnership and close collaboration with their stakeholders and communities, all of whom have different understandings, aims and organisational abilities (Cox et al. 2013, Potts et al. 2016). In this context, it is therefore not sufficient that the NRM organisation itself should learn; learning must extend throughout all the actors in the NRM system (Wallis et al. 2013).

Despite the importance attributed to learning in the NRM literature (Dietz et al. 2003), findings elicited by the validation of the conceptual model indicate that a learning 'culture' is still not a key characteristic of NRM organisations. This situation calls for NRM organisations to actively develop a 'culture that values reflection, learning, experimentation, complexity and diversity' (Lockwood et al. 2009), and that it is not afraid of failure (Chapman 2004). This entails a shift from an organisation that uses monitoring and evaluation to assess external outcomes and generate 'lessons learnt', to a learning organisation that promotes learning about the role, functions and relationships of the organisation in the context of the broader socio-ecological system (Goold 2006). However, such a shift would require broad support from stakeholders and related organisations including funding bodies as current governance and accountability measures do not support experimentation and may be averse to failure (Adams et al. 2007).

Notably, best practice was highlighted by planners as being both the result of multiple learning processes and the motivator to start and sustain collaborations with stakeholders, researchers and the community of practice. Additionally, knowledge translation was identified as having the highest potential among learning processes to achieving NRM outcomes in the long-term. Hence, there is a clear role that bridging organisations (Crona et al. 2012) could play in organisational learning if learning 
becomes an explicit goal of collaborations. Improved documentation of regional and cross-regional approaches is also needed to facilitate cross-regional sharing of the learning. Working with stakeholder groups who already have cross-regional linkages provides additional perspectives and network linkages that can significantly contribute to improved organisational capacity to deliver decision-making outcomes, connectivity between different organisations, stakeholders and governance structures, and application of knowledge in decision-making processes (Potts et al. 2016).

\section{Reinventing practice}

Several authors have suggested that business as usual approaches will not be sufficient, and that NRM organisations need to adopt approaches that increase the resilience and adaptive capacity of social-ecological systems, including recognition of the role of the organisations themselves in the system (Gooch et al. 2009). NRM involves stakeholders including funding bodies and multiple scales of government, and therefore is subject to governance problems which need be acknowledged when considering learning processes (Vella et al. 2015, Jacobson et al. 2014). Through the validation of the conceptual model planners indicated that there are two typical learning processes to NRM that are failing to contribute to NRM outcomes: program auditing, and the very core of the adaptive management cycle - the MERI process. Both processes have become grounded on excessive reporting per se rather than based on the actual NRM outcomes sought to be achieved. However, planners also noted that their organisations are relatively open to adaptive co-management approaches, and the high potential of knowledge translation in achieving NRM outcomes in the long-term. Two key messages can be drawn from these findings.

Firstly, NRM practice driven by ‘accountability' and organisational 'efficiency' attributes needs to change as current processes driving it are failing to deliver NRM outcomes. 
Secondly, a culture of reflection and ongoing learning needs to be encouraged in the NRM sector, which could be enacted through a coherent, holistic learning strategy, led by the NRM organisation. However, for NRM organisations to effectively implement the suggestions above, they need support from a broader governance system. They need to build their capacity for adaptive or transformative governance and management (Mitchell et al. 2014). One possible effective structure is that of polycentric governance system (Pahl-Wostl 2009), where modular systems with differently sized governance units with different purpose, organisation and spatial location interact to form together a largely self-organised governance regime. Polycentric governance systems are characterized by many degrees of freedom at different levels. Multi-level governance in polycentric systems implies that decisionmaking authority is distributed in a nested hierarchy and does not reside at one single level, neither top (only highest level government enforcing decisions), nor medium (only states enforcing decisions beneficial for their region without considering others), nor individuals with complete freedom to act or being connected in a market structure only. From a normative point of view it is of interest that polycentric systems are assumed to have a higher ability to adapt to a changing environment and to be less affected in their integrity by sudden changes or failure in parts of the system (Ostrom 2010, Pahl-Wostl 2009).

This would include learning by the entire NRM institution (including the funding bodies, NRM organisations and stakeholders), and focus on improved governance mechanisms. It would incorporate learning across regions and organisational learning of the funding bodies and stakeholders as well as NRM organisations. 


\section{Conclusions}

This paper tested a conceptual model with NRM planners to identify linkages between learning processes and NRM outcomes. The conceptual model provided a necessarily simplified version of events, and in reality the distinctions between the different learning situations may not be so clear-cut. However, it was useful to assist NRM planners in assessing the range of learning activities that they undertake in relation to the relevant learning goals and outcomes they are bound to deliver, and to help them in identifying processes and practices that promote specific types of learning.

Supporting adaptive learning approaches in NRM is critical to allow organisations to build their adaptive capacity to deal with increasing uncertainty and change. This includes developing planner's capacity to exchange, access and apply existing and new knowledge to support evidence-based decision-making; thereby minimising the negative impacts of being over-stretched to deliver better environmental outcomes whilst managing increased land use pressures and competition for natural resources (not to mention decreased funding sources and emergence of inherent competition between different organisations).

There are two key insights from this research that may also be relevant to planners working in other sectors outside NRM. This includes, but is not limited to, less resourced government agencies with similar shortages of expertise albeit having to manage increased land use pressures, rapid urbanisation and politically charged as opposed to evidence-based decisions. Firstly, the research confirmed that there is no learning culture embedded in the NRM organisations and this could also be the case of other organisations such as government agencies with a planning portfolio. However, developing and implementing best practice was found to be a result of learning. This points to the need for organisations to clearly articulate learning 
structures within their management processes to ensure their practitioners can perform their jobs to the best of their abilities. Additionally, these learning structures need to facilitate the flow of information and (tacit) knowledge from previous to new staff members considering the high rate of staff churn observed.

Secondly, the increased corporatisation of government agencies has led to a rise in auditing and reporting activities that were found to be inhibiting rather than enabling learning to occur, including in the adaptive management cycle (MERI). This is an important finding that has relevance for the broader planning practice because there has been ongoing interest in embedding adaptive management in both plan making and plan implementation endeavours. In particular, it is fundamental to pay attention to the reporting component of the adaptive management cycle so as to ensure it doesn't replace the very essence of reflection that needs to drive the process. Reporting for the sake of reporting without entailing reflection will certainly impede learning and, more importantly, best practice to be implemented.

\section{References}

Adams, Carol A., and Patty McNicholas. 2007. Making a difference: Sustainability reporting, accountability and organisational change. Accounting, Auditing \& Accountability Journal 20 (3):382-402.

Ardichvili, Alexander, Vaughn Page, and Tim Wentling. 2003. Motivation and barriers to participation in virtual knowledge-sharing communities of practice. Journal of knowledge management 7 (1):64-77.

Armitage, Derek, Melissa Marschke, and Ryan Plummer. 2008. Adaptive comanagement and the paradox of learning. Global Environmental Change 18 (1):86-98.

Australian Government. 2014. "Regional NRM organisations." Australian Government National Landcare Programme Accessed 14/01/2015. http://www.nrm.gov.au/regional/regional-nrm-organisations.

Baird, Julia, Ryan Plummer, Constanze Haug, and Dave Huitema. 2014. Learning effects of interactive decision-making processes for climate change adaptation. Global Environmental Change 27 (0):51-63.

Berkes, Fikret. 2009. Evolution of co-management: Role of knowledge generation, bridging organizations and social learning. Journal of Environmental Management 90 (5):1692-1702. 
Bielak, Alex T, Andrew Campbell, Shealagh Pope, Karl Schaefer, and Louise Shaxson. 2008. From science communication to knowledge brokering: the shift from 'science push'to 'policy pull'. In Communicating science in social contexts, 201-226. Springer.

Chapman, Jake. 2004. System failure: Why governments must learn to think differently: Demos.

Clayton, Helena, Steve Dovers, and Paul Harris. 2011. Synthesis of broad issues and opportunities: Document I. Canberra: HC Coombs Policy Forum-Fenner School of Environment and Society NRM initiative, The Australian National University.

Cook, William, Séverine van Bommel, and Esther Turnhout. 2016. Inside environmental auditing: effectiveness, objectivity, and transparency. Current Opinion in Environmental Sustainability 18:33-39.

Cox, M., S. Serrao-Neumann, K. Vella, M. Sano, and D. Low Choy. 2013. Analysis of the Needs of the East Coast Cluster Regional Natural Resource Management Bodies in Relation to Planning for Climate Change Adaptation. Climate Change Adaptation for Natural Resource Management in East Coast Australia Project, Griffith University.

Crona, Beatrice I., and John N. Parker. 2011. Network Determinants of Knowledge Utilization: Preliminary Lessons From a Boundary Organization. Science Communication 33 (4):448-471.

Crona, Beatrice I., and John N. Parker. 2012. Learning in Support of Governance: Theories, Methods, and a Framework to Assess How Bridging Organizations Contribute to Adaptive Resource Governance. Ecology and Society 17 (1).

Cundill, Georgina, and Christo Fabricius. 2009. Monitoring in adaptive comanagement: Toward a learning based approach. Journal of Environmental Management 90 (11):3205-3211.

Cvitanovic, C., J. McDonald, and A. J. Hobday. 2016. From science to action: Principles for undertaking environmental research that enables knowledge exchange and evidence-based decision-making. Journal of Environmental Management 183, Part 3:864-874.

Davidson-Hunt, Iain, and Fikret Berkes. 2003. Learning as You Journey: Anishinaabe Perception of Social-ecological Environments and Adaptive Learning. Ecology and Society 8 (1).

De Geus, Arie P. 1988. Planning as learning: Harvard Business Review.

Dietz, Thomas, Elinor Ostrom, and Paul C. Stern. 2003. The Struggle to Govern the Commons. Science 302 (5652):1907-1912.

Gooch, Margaret, and Jeni Warburton. 2009. Building and Managing Resilience in Community-Based NRM Groups: An Australian Case Study. Society \& Natural Resources 22 (2):158-171.

Goold, Liz. 2006. Working with Barriers to Organisational Learning. Bond Briefing Paper.

Government, NSW. 2013. Local Land Services Act 2013 No 51.

Hachmann, Verena. 2008. Promoting Learning in Transnational Networks. disP - The Planning Review 44 (172):11-20.

Hasselman, Lyndal. 2016. Adaptive management; adaptive co-management; adaptive governance: what's the difference? Australasian Journal of Environmental Management:1-16. 
Hassink, Robert, and Arnoud Lagendijk. 2001. The dilemmas of interregional institutional learning. Environment and Planning C 19 (1):65-84.

Hobday, Alistair J., Veronica A. J. Doerr, Nadine A. Marshall, Christopher Cvitanovic, and Lilly Lim-Camacho. 2017. Adapting to climate change: the role of organisational personalities in natural resource management. Regional Environmental Change.

Hulme, Philip E. 2014. Bridging the knowing-doing gap: know-who, know-what, knowwhy, know-how and know-when. Journal of Applied Ecology:n/a-n/a.

Jacobson, C., K. F. D. Hughey, A. J. J. Lynch, M. Nursey-Bray, M. O'Connell, P. G. Munro, K. Vella, D. Whiley, S. Dovers, and R. W. Carter. 2014. Twenty years of pacifying responses to environmental management. Australasian Journal of Environmental Management 21 (2):143-174.

Levesque, Vanessa R., Aram J. K. Calhoun, Kathleen P. Bell, and Teresa R. Johnson. 2016. Turning Contention into Collaboration: Engaging Power, Trust, and Learning in Collaborative Networks. Society \& Natural Resources:1-16.

Lockwood, Michael, Julie Davidson, Allan Curtis, Elaine Stratford, and Rod Griffith. 2009. Multi-level Environmental Governance: lessons from Australian natural resource management. Australian Geographer 40 (2):169-186.

Lonsdale, Kate. 2012. Beyond tools: building learning organisations to adapt to a changing climate. Victorian Centre for Climate Change Adaptation Research.

Michaels, Sarah. 2009. Matching knowledge brokering strategies to environmental policy problems and settings. Environmental Science \& Policy 12 (7):994-1011.

Mitchell, Michael, Rod Griffith, Paul Ryan, Greg Walkerden, Brian Walker, Valerie A. Brown, and Sandy Robinson. 2014. Applying Resilience Thinking to Natural Resource Management through a "Planning-By-Doing" Framework. Society \& Natural Resources 27 (3):299-314.

Muro, M., and P. Jeffrey. 2008. A critical review of the theory and application of social learning in participatory natural resource management processes. Journal of Environmental Planning and Management 51 (3):325-344.

Newig, Jens, Dirk Günther, and Claudia Pahl-Wostl. 2010. Synapses in the Network: Learning in Governance Networks in the Context of Environmental Management. Ecology \& Society 15 (4).

Ostrom, Elinor. 2010. Beyond Markets and States: Polycentric Governance of Complex Economic Systems. American Economic Review 100 (3):641-672.

Pahl-Wostl, C. 2007. The implications of complexity for integrated resources management. Environmental Modelling and Software 22 (5):561-569.

Pahl-Wostl, Claudia. 2009. A conceptual framework for analysing adaptive capacity and multi-level learning processes in resource governance regimes. Global Environmental Change 19 (3):354-365.

Patterson, James J. 2016. Exploring Local Responses to a Wicked Problem: Context, Collective Action, and Outcomes in Catchments in Subtropical Australia. Society \& Natural Resources 29 (10):1198-1213.

Plummer, Ryan. 2009. The Adaptive Co-Management Process: an Initial Synthesis of Representative Models and Influential Variables. Ecology and Society 14 (2).

Plummer, Ryan, and Derek Armitage. 2007. A resilience-based framework for evaluating adaptive co-management: Linking ecology, economics and society in a complex world. Ecological Economics 61 (1):62-74. 
Plummer, Ryan, Beatrice Crona, Derek R. Armitage, Per Olsson, Maria Tengö, and Olga Yudina. 2012. Adaptive Comanagement: a Systematic Review and Analysis. Ecology and Society 17 (3).

Potts, Ruth, Karen Vella, Allan Dale, and Neil Sipe. 2016. Evaluating Governance Arrangements and Decision Making for Natural Resource Management Planning: An Empirical Application of the Governance Systems Analysis Framework. Society \& Natural Resources 29 (11):1325-1341.

Reason, P, and H Bradbury. 2006. Handbook of Action Research London: SAGE.

Reed, Mark S, Anna C Evely, Georgina Cundill, Ioan Fazey, Jayne Glass, Adele Laing, Jens Newig, Brad Parrish, Christina Prell, and Chris Raymond. 2010. What is social learning? Ecology \& Society 15 (4).

Reed, Mark S., Evan D. G. Fraser, and Andrew J. Dougill. 2006. An adaptive learning process for developing and applying sustainability indicators with local communities. Ecological Economics 59 (4):406-418.

Reed, Mark S., Anil Graves, Norman Dandy, Helena Posthumus, Klaus Hubacek, Joe Morris, Christina Prell, Claire H. Quinn, and Lindsay C. Stringer. 2009. Who's in and why? A typology of stakeholder analysis methods for natural resource management. Journal of Environmental Management 90 (5):1933-1949.

Robins, Lisa, and Stephen Dovers. 2007. NRM Regions in Australia: the 'Haves' and the 'Have Nots'. Geographical Research 45 (3):273-290.

Ryan, S, K Broderick, Y Sneddon, and K Andrews. 2010. Australia's NRM governance system: Foundations and principles for meeting future challenges. Canberra: Australian Regional NRM Chairs. Canberra: Australian Regional NRM Chairs.

Shaxson, L, T Bielak, I Ahmed, D Brien, B Conant, C Fisher, E Gwyn, L Klerkx, A Middleton, and S Morton. 2012. Expanding our understanding of $K^{*}(K T, K E$, $\mathrm{KTT}, \mathrm{KMb}, \mathrm{KB}, \mathrm{KM}$, etc.). A concept paper emerging from the $\mathrm{K}^{*}$ conference held in UNU-INWEH Hamilton, ON.

Smith, T. F., R. W. Carter, D. C. Thomsen, G. Mayes, M. Nursey-Bray, G. Whisson, R. Jones, S. Dovers, and K. O'Toole. 2009. Enhancing Science Impact in the Coastal Zone through Adaptive Learning. Journal of Coastal Research:13061310.

Tennent, Rebeka, and Stewart Lockie. 2012. Vale Landcare: the rise and decline of community-based natural resource management in rural Australia. Journal of Environmental Planning and Management 56 (4):572-587.

van der Wal, Merel, Joop De Kraker, Astrid Offermans, Carolien Kroeze, Paul A. Kirschner, and Martin van Ittersum. 2014. Measuring Social Learning in Participatory Approaches to Natural Resource Management. Environmental Policy and Governance 24 (1):1-15.

Vella, Karen, Neil Sipe, Allan Dale, and Bruce Taylor. 2015. Not Learning from the Past: Adaptive Governance Challenges for Australian Natural Resource Management. Geographical Research 53 (4):379-392.

Viegas, Cláudia V., Alan Bond, José Luis Duarte Ribeiro, and Paulo Maurício Selig. 2013. A review of environmental monitoring and auditing in the context of risk: unveiling the extent of a confused relationship. Journal of Cleaner Production 47:165-173.

Vogel, Norbert. 2013. Analysis of Performance Excellence Reviews of Regional NRM Organisations. AKM Group. 
Wallis, Philip, Karyn Bosomworth, Hartmut Fünfgeld, Sophie Millin, Alianne Rance, and Kate Lonsdale. 2013. Climate Change Adaptation in the Natural Resource Management Sector of Victoria: A literature review and analysis of institutional context and organisational needs for adaptation. Victorian Centre for Climate Change Adaptation Research.

Wenger, Etienne. 1998. Communities of practice: Learning, meaning, and identity: Cambridge university press.

Wenger, Etienne. 2000. Communities of practice and social learning systems. Organization 7 (2):225-246. 